\title{
Tratamiento electoral en Ecuador: estudio sobre la exposición mediática de los candidatos a alcalde en las elecciones de Manta (2014)
}

\author{
MHCJ no 6 | Año 2015 \\ Artículo no 4 (65) \\ Páginas 69 a 90 \\ mhjournal.org
}

\author{
Juan Pablo Trámpuz | juanptrampuz@hotmail.com \\ Universidad Laica Eloy Alfaro de Manabí \\ Daniel Barredo Ibáñez | daniel.barredo@uleam.edu.ec \\ Universidad Laica Eloy Alfaro de Manabí
}

\section{Palabras clave}

Tratamiento electoral; exposición mediática; elecciones Ecuador; análisis de contenido

Sumario

1. Introducción. 1.2. Ecuador y la importancia del uso político de Internet

\section{Methodology. 2.1. Sample}

3. Results. 3.1. Manual analysis of content. 3.2. Computerized content analysis 3.3. Indepth interviews

4. Conclusiones

5. Bibliografía

6. Notas

\section{Resumen}

El presente trabajo de investigación busca analizar la exposición mediática de los candidatos a la alcaldía de Manta de 2014 en los contenidos digitales de los medios escritos de la provincia de Manabí (Ecuador), para lo cual se realizó un análisis de contenido manual e informatizado de las notas emitidas durante la temporada oficial de la campaña electoral (7 de enero hasta el 20 de febrero 2014) en los periódicos El Mercurio, La Marea, El Manaba y El Diario; así como entrevistas a cuatro de los seis candidatos a alcalde. El análisis aborda, además de la exposición mediática, aspectos como los géneros, secciones y temas prioritarios, logrando identificar aspectos relevantes del discurso periodístico que se generó en torno al ambiente electoral que se vivió en Manta. Según los datos de los análisis, y las percepciones de los candidatos trianguladas con las técnicas cuantitativas, subrayamos el incumplimiento en estos medios de la Ley Orgánica de Comunicación, al no conceder a todos los protagonistas de dicho proceso electoral un trato equitativo.

\section{Forma de citar este artículo en las bibliografías}

Juan Pablo Trámpuz y Daniel Barredo Ibáñez (2015): “Tratamiento electoral en ecuador: estudio sobre la exposición mediática de los candidatos a alcalde en las elecciones de Manta (2014)”, en Miguel Hernández Communication Journal, nº, páginas 69 a 90. Universidad Miguel Hernández, UMH (Elche-Alicante). Recuperado el _ de de $20 \_$de: http://mhjournal.org/[ink del artículo] 


\section{Electoral treatment in Ecuador: study on media exposure of mayoral candidates in the elections of Manta (2014)}

MHCJ no 6 | Year 2015

Paper no 4 (65)

Pages 69 to 90

mhjournal.org
Juan Pablo Trámpuz | juanptrampuz@hotmail.com

Universidad Laica Eloy Alfaro de Manabí

Daniel Barredo Ibáñez | daniel.barredo@uleam.edu.ec

Universidad Laica Eloy Alfaro de Manabí
Keywords

Electoral Treatment; Media exposure; Elections Ecuador; Content analysis

Sumary

1. Introduction. 1.2. Ecuador and the importance of political use of the Internet

2. Methodology.

3. Results.

4. Conclusions

5. Bibliography.

6. Notes.

\begin{abstract}
The present research analyzes the media exposure of candidates for mayor of Manta, 2014, in digital content of the written media in the province of Manabí (Ecuador). There has been developed a manual and computerized analysis of content of the news published during the official electoral campaign (January 7 to February 20, 2014) by the newspapers El Mercurio, La Marea, The Manaba and El Diario; also, we have interviewed four of the six candidates for mayor. The analysis, in addition to media exposure, addresses issues such as gender, sections and priority issues, identifying relevant aspects of journalistic discourse that was generated around the electoral environment that lived in Manta. According to data analysis, and perceptions of the candidates triangulated with quantitative techniques, stress failure in these media Communications Law by failing to grant all the protagonists of the electoral process fairly.
\end{abstract}

How to cite this paper in bibliographies

Juan Pablo Trámpuz y Daniel Barredo Ibáñez (2015): “Electoral treatment in Ecuador: study on media exposure of mayoral candidates in the elections of Manta (2014)", en Miguel Hernández Communication Journal, n6, pages 69 to 90. Universidad Miguel Hernández, UMH (Elche-Alicante). Recuperado el de de 20 


\section{Introducción: reflexiones sobre la opinión pública contemporánea ${ }^{1}$}

Floyd Allport (1937) señaló que el individuo sólo tiene tres maneras de realizar deducciones sobre las opiniones y puntos de vista predominantes entre la población: la prensa, el rumor y la proyección social. De estas tres opciones, solamente una está plenamente establecida e identificada: los medios de comunicación masivos.

Los medios de comunicación ocupan un espacio protagónico en las sociedades contemporáneas; es por ello que incluso se menciona a una sociedad de los media (Muñoz, 2005), configurada en parte por las instituciones mediáticas. Trabajos más recientes, como los de Oller y Barredo (2012) o el de Barredo, Oller y Buenaventura (2013), señalan la aparición de una sociedad identificada por patrones de activación social, donde los ciudadanos tienden a cogestionar o autogestionar la esfera simbólica que en momentos anteriores estaba monopolizada por los medios de comunicación; si bien, y a pesar de estas transformaciones, dichos medios continúan concentrando la mayor parte de la repercusión, quizá porque conservan todavía parte de la legitimidad de las épocas anteriores. Una influencia que, según McQuail (2000), pueden desempeñar los medios en función de cuatro roles vinculados a la cotidianidad:

1. Son un recurso de poder, al tener la capacidad de ser utilizados como un instrumento de presión, control e innovación en la sociedad.

2. Tienden a representar la esfera donde se desarrollan muchos asuntos de la vida pública.

3. Son una fuente de definición e imágenes de la realidad social.

4. Y originan el establecimiento de patrones de normalidad, tanto empírica como subjetivamente.

En las sociedades democráticas, el rol de los medios de comunicación es central en los procesos electorales, como apunta Ortega (2011), debido sobre todo a su protagonismo como difusores de instrumentos de persuasión, y a su posicionamiento central dentro de los debates públicos. Pese a las aportaciones teóricas y a diversos estudios, siempre queda latente la interrogante que plantea qué influencia tienen los medios de comunicación de masas sobre los individuos. Es esta una cuestión que ha intrigado a varios estudiosos de las Ciencias Sociales, principalmente luego del desarrollo de hechos acaecidos durante el siglo XX, donde la comunicación jugó un rol protagónico a través de la propaganda, como las dos guerras mundiales o la conformación de la Unión Soviética y la Alemania Nazi (McQuail, 2000). Precisamente en esos periodos altamente simbólicos, se produjo un incremento del uso de los mass media, con la siguiente atribución de su capacidad para moldear la opinión pública ${ }^{2}$, y el rol de estos canales para modificar la percepción de las masas (De Fleur y Ball-Rokeach, 1988). Es decir, se le otorgó a los medios una doble función de notable importancia: la de influir en el pensamiento del individuo en sociedad, y la de llamar la atención sobre determinados temas, estableciendo una especie de agenda social en base a los criterios de los medios de comunicación.

A pesar de esta afirmación generalizadora -que tomó fuerza a mediados del siglo XX-, hoy se sabe que las audiencias no reaccionan como una masa inerte ante los mensajes que recibe desde los medios de comunicación social (Lull, 1997), pues cada persona tiende a decodificar la 
información según sus capacidades cognitivas y sus respectivos contextos culturales; sin embargo, este mismo autor los cataloga como uno de los más eficaces transmisores de ideología, ratificando así su influencia en la sociedad, independientemente de los efectos que puedan generarse. En este contexto, se acuñó en torno a las instituciones mediáticas el término cuarto poder (Alonso y Santana, 2000), lo que se entendió como una especie de contrapeso a los tres poderes ya establecidos en los Estados democráticos: ejecutivo, legislativo y judicial (Ramonet, 2011), fundamentándose en el deber cívico del periodista, algo que en la mayoría de los casos pasó a un segundo plano frente a la comercialización de los medios (Thompson, 1996). No pasó mucho tiempo hasta que el cuarto poder perdió su sentido, y alejándose de su aspecto fiscalizador, los medios tomaron un rol más importante para sí mismos: "el espacio donde se crea el poder" (Castells, 2009: 262). La afirmación de este sociólogo español tiene su origen en la idea de que aquella esfera pública a la que Habermas (1986) señalaba como el espacio mediador entre el Estado y la sociedad, ha sido absorbida por los medios de comunicación (Ortega, 2011); con lo cual, los mass media se convirtieron en el lugar donde las sociedades ventilan los aspectos de interés público, generando debates, opiniones y cuestionamientos al respecto, decidiéndose así las relaciones de poder.

Uno de los ámbitos de mayor interés público en las sociedades democráticas es la política, un aspecto que también ha sido acaparado por los medios de comunicación, transformándola y adaptándola a su lógica de espectáculo y superficialidad, donde se priorizan aspectos como las relaciones públicas, el montaje de shows mediáticos, y la fabricación o manipulación de la opinión pública (Fraser, 1992). Es por ello que Castells (2009: 261) afirma que actualmente "la política es fundamentalmente mediática", lo que significa que la misma se hace en y a través de los medios de comunicación. Esta aseveración es señalada como el origen de muchos cambios en la política actual (Ortega, 2011), donde cada vez tienen más espacio figuras como futbolistas, presentadores de televisión y cantantes (El Universo, 2012, 11 de noviembre), por encima de personas que, pese a tener aptitudes, no encajan con facilidad en el discurso mediático, y por ende será más difícil incluirlos en la esfera pública.

En Ecuador, al igual que en otras partes del mundo, varios medios de comunicación han sido señalados como actores políticos (Abad, 2011; Isch, 2012), asumiendo roles de oposición y apoyo a determinados sectores o movimientos. Este protagonismo de los medios en la arena política se acentúa durante las elecciones, momentos que son considerados aparentemente como lo fundamental de la democracia (Ruiz, 2005), donde prima el interés de los grupos de poder por influenciar en la opinión de los votantes a fin de ganar su voluntad.

La influencia que proviene desde el poder político a través de los medios, no apunta necesariamente a la capacidad de reflexión de los electores, sino más bien a aspectos emotivos que se construyen con el abarrotamiento de mensajes amplificados por los medios de comunicación, ya que: " $<\ldots>$ el objetivo de las campañas electorales es precisamente no dejar tiempo para reflexionar hasta el momento de la decisión. Intentan, por el contrario, sacudir a la opinión pública" (Noelle-Neumann, 1995: 171).

Una de las formas de agitar la opinión pública es fabricar falsas realidades, con lo cual se aspira poner en marcha el efecto del carro ganador, que consiste en que otros electores se sumen a la tendencia que aparentemente tiene mayores posibilidades de vencer en la lid electoral (NoelleNeumann, 1995). Goffman y Rodríguez (2006: 89) definen a la fabricación de falsas realidades como "el esfuerzo deliberado de uno o más individuos para manejar una actividad de modo que induzca a otros a formarse una creencia falsa de lo que está sucediendo", por lo que se 
deduce que esta acción mediática es premeditada, y se desarrolla ya sea en solitario o en alianza con otros medios, actores políticos y sociales, que logren sintonizar intereses en común.

\subsection{Ecuador y la importancia del uso político de Internet}

Ecuador es un país que podría ser descrito, en primer lugar, por la brecha digital; si, en 2013, solo cuatro de cada diez ecuatorianos podían acceder a Internet (Ministerio de Telecomunicaciones, 2014, 19 de mayo), estudios como el de Barredo y Silva (2014) han identificado la baja participación que se da en el ciberespacio de ese país, motivada tanto por un desconocimiento de las rutinas de interacción ancladas a lo digital, como por -en generalla escasa participación propia de los ciudadanos en los medios convencionales o en la esfera política. Con todo, algunos trabajos previos han subrayado la importancia que tienen las redes sociales en, por ejemplo, las contiendas electorales (León, 2007; Rivera Rogel, 2014), quizá porque precisamente en las redes se concentra una minoría social que tiene un fuerte impacto en el panorama simbólico ecuatoriano. Hasta un tercio de los encuestados en dos ciudades representativas del país -Quito y Riobamba- aseguraron otorgar una mayor credibilidad a los contenidos de las redes sociales que a los contenidos de los medios de comunicación convencionales (Barredo, Pérez Zúñiga, Vivas y Fernández, 2015), unos datos que contrastan severamente con las cifras expuestas al inicio de este apartado.

La presente investigación se centró en la campaña política por la alcaldía de Manta en el año 2014, que contó con la participación de seis candidatos: Jaime Estrada, por la coalición Alianza País-Unidad Primero; Jorge Zambrano, por el movimiento CREO; María Zambrano de Gando, SUMA; Leonardo Cárdenas, Alianza Cívica Ciudadana; Juan Carlos Álvia, movimiento Machete; y Gustavo Andrade, partido Socialista (eleccionesenecuador.com, s. f. ).

Los recorridos, propuestas, críticas y respaldos a los candidatos fueron difundidos por los principales medios digitales escritos provinciales con circulación en esta ciudad, que según el portal www.alexa.com ${ }^{3}$ son Medios Ediasa (El Diario Manabita y La Marea), el Mercurio, el Manaba y la Gente de Manabí.

En concordancia con lo establecido en el Art. 115 de la Constitución de la República del Ecuador, el Art. 203 de la Ley Orgánica Electoral y de Organizaciones Políticas de la República de Ecuador, y el Art. 72 de la Ley Orgánica de Comunicación, nos planteamos como hipótesis:

Determinar si la emisión de contenidos digitales difundidos por los medios de comunicación escritos de Manabi durante la campaña electoral por la alcaldía de Manta en 2014, favoreció la exposición mediática de determinados candidatos.

El contraste de esta hipótesis permitirá establecer interesantes pautas sobre un contexto -el de Manabí, Ecuador, relacionado con el uso político de las redes sociales- que a pesar de su significancia para el conjunto de la población de esa región, ha sido muy poco investigado. 


\section{Metodología}

Este trabajo, de carácter exploratorio, ha trazado un enfoque multimodal para recoger la mayor cantidad posible de aspectos sobre el periodo electoral estudiado. Dentro de las técnicas cuantitativas, se han practicado sendos análisis de contenido -manual e informatizado- sobre los contenidos alusivos a los candidatos a la alcaldía de Manta en los medios El Diario, La Marea, El Mercurio, y El Manaba. El periodo escogido fue desde el 7 de enero al 20 de febrero de 2014, es decir, el momento álgido de las elecciones municipales que se celebraron el 23 de febrero del mismo año. Este periodo de monitoreo tuvo en cuenta la recomendación del Consejo Nacional Electoral -máximo organismo del país en temas electorales- de fijar este plazo ( $7-20$ de febrero) como las fechas límites para hacer campaña política por parte de los distintos candidatos en todo el país 4 .

La elección de la prensa digital responde a dos realidades. La primera es la creciente importancia de los medios digitales en el contexto mundial (Boczkowski, 2006) y nacional ${ }^{5}$, lo que ha generado una serie de investigaciones en torno a estos medios en nuestro país (Rivera Rogel, 2010; 2011; y Rivera Costales, 2013), entre otros. La segunda, es la relativa facilidad con la que se puede acceder a los contenidos de los medios digitales para efectuar análisis complejos vinculados al desarrollo de técnicas informáticas.

Los medios El Diario, La Marea, El Mercurio, y El Manaba, fueron escogidos por brindar una constante cobertura al proceso electoral en la ciudad de Manta, además de por contar con ediciones digitales disponibles de manera actualizada. En primera instancia se planteó incluir en el análisis al medio digital La Gente de Manabí; sin embargo, al no contar con archivos PDF y, principalmente, al ser la mayoría de sus contenidos boletines de prensa de campaña de los dos candidatos que tuvieron una maquinaria comunicacional, se excluyó del estudio.

$\mathrm{Al}$ iniciar el estudio, se constató que las noticias de estos cuatro medios (principalmente El Mercurio y El Manaba) no registraron constancia de difusión en sus páginas web, por lo cual, se recurrió a las versiones impresas digitalizadas en formato PDF; teniendo así un análisis más cercano a la difusión de estos medios durante la campaña por la alcaldía de Manta 2014.

El tipo de investigación desarrollado es básica polietápica, que alude a un estudio realizado en función de varios pasos o etapas, donde el monitoreo y la estadística que se deriven juegan un papel preponderante en medir el grado de relación que existe entre las variables, utilizando diferentes instrumentos y software para el desarrollo de los acontecimientos. Las dos técnicas cuantitativas empleadas fueron el análisis de contenido manual y el análisis de contenido informatizado.

Berelson (1952) definió al análisis de contenido como una técnica de investigación para la descripción objetiva, sistemática y cuantitativa del contenido manifiesto de la comunicación. Estas tres características han sido descritas por Abela (2002: 2):

Objetividad: Usando técnicas que puedan ser utilizadas por otros investigadores para verificar los resultados obtenidos.

Sistematización: Uso de pautas ordenadas durante el desarrollo del análisis. 
Cuantificable: Que se pueda cifrar numéricamente la información.

El análisis de contenido manual se operó mediante una ficha de análisis, siendo esta una herramienta que sirve para organizar y recopilar analíticamente un conjunto de datos, y que se puede diseñar de acuerdo a las características de cada investigación (Igartua y Muñiz, 2012). Como parte de esta técnica se consideraron varios apartados que se ilustran en los resultados.

El análisis de contenido informatizado es una técnica que permite examinar grandes cantidades de material textual a través del uso de herramientas informáticas (Barredo, 2012), por lo cual se consideró esta técnica para complementar el análisis de contenido manual; siendo de gran utilidad para identificar y elaborar listas de términos clave encontrados en las unidades analizadas.

Para objeto de este análisis, se tomó como programa base el Concordance $e^{6}$, desarrollado a partir de 1999 por el profesor Robert J. Watt, debido a la facilidad con que permite contar palabras clave, enlistarlas, priorizarlas, y al mismo tiempo mostrarlas en su contexto.

Como parte del análisis se realizaron dos tipos de exclusiones, mismos que han sido aplicados emulando un estudio pionero en este ámbito, como lo es El tabú de la expresividad real. Análisis del tratamiento informativo del rey Juan Carlos I en ABC.es y ELPAÍS.com (2009 - 2011) de Daniel Barredo (2012).

La primera es la exclusión mecánica, que considera lo siguiente:

Palabras que no aportan una información diferenciada como la firma del periodista, redacción, fecha, etc.

Y las exclusiones lingüísticas:

Palabras sin significado propio (preposiciones, demostrativos, adverbios, artículos determinados o indeterminados, conjunciones, etc.).

Verbos como ser, estar, haber y hacer.

Otras palabras que no generan mayor importancia para el objeto de estudio como números, nombres propios de otros cantones, etc.

Además, se recodificaron ciertos términos considerados clave para que Concordance pueda contabilizar los términos como tal, y no separar las palabras, dificultando su correcta interpretación, entre ellos:

Los nombres de los candidatos a la alcaldía de Manta 2014: GustavoAndrade por Gustavo Andrade.

Cuando los medios hicieron alusión a un candidato únicamente por su primer apellido, ejemplo: JaimeEstrada por Estrada.

Los nombres de otros personajes de especial relevancia dentro de la campaña política como el vicepresidente, ministros, candidato a prefecto; ejemplo: JorgeGlas por Jorge Glas. 
Términos clave como plan hidrosanitario, terminal terrestre, paso elevado, etc.; ejemplo: PlanHidrosanitario por Plan Hidrosanitario.

Movimientos y partidos políticos, ejemplo: AlianzaPaís por Alianza País.

En cuanto a la tabulación y procesamiento de la información, se realizó en el programa SPSS (Paquete Estadístico para las Ciencias Sociales) versión 20.0.0.0, desarrollado en la Universidad de Chicago. La selección del SPSS para el análisis de los datos obedeció a que el mismo es uno de los programas más difundidos y sencillos de utilizar (Hernández Sampieri, Fernández y Baptista, 2010).

A fin de cumplir con el objetivo de conocer la perspectiva de los candidatos a la alcaldía de Manta, respecto a la difusión brindada por los medios de comunicación a la campaña 2014, se recurrió a una de las técnicas ampliamente utilizadas en la investigación social como lo es la entrevista en profundidad, ya que este instrumento permite obtener de los entrevistados información y elementos de reflexión muy ricos y llenos de matices (Quivy y Van Campenhoudt, 1992).

\subsection{Muestra}

Se analizaron en total 181 noticias, de las cuales fueron publicadas 73 en El Mercurio, 53 en La Marea, 30 en El Manaba, y 25 en El Diario Manabita, identificadas por la alusión a uno o más candidatos a la alcaldía de Manta 2014 durante el periodo temporal escogido.

Para las entrevistas, se buscó aplicar esta técnica a los seis candidatos, de los cuales únicamente cuatro aceptaron participar: Juan Carlos Alvia, Gustavo Andrade, Leonardo Cardenas y María Zambrano de Gando.

\section{Resultados}

\subsection{Análisis de contenido manual}

\subsubsection{Géneros periodísticos}

Tras efectuar un análisis comparativo entre los cuatro medios de comunicación estudiados, encontramos que los mismos mantuvieron ciertas tendencias como fue el uso mayoritario de la noticia y la escasa presencia de géneros de opinión e investigativos, un hecho que podía redundar en la escasa profundización interpretativa sobre las propuestas de los candidatos a alcalde. 
FIG. 1 Géneros periodísticos en las unidades de análisis de los medios escritos locales durante las elecciones a la alcaldía de Manta (Ecuador) en 2014

\begin{tabular}{|l|l|l|l|l|l|l|l|l|l|l|l|}
\hline \multirow{2}{*}{ Género } & \multicolumn{2}{|l|}{ E1 Mercurio } & \multicolumn{2}{l}{ La Marea } & \multicolumn{2}{l}{ E1 Manaba } & \multicolumn{2}{l|}{ E1 Diario } & \multicolumn{2}{l|}{ Total } \\
\cline { 2 - 14 } & $\mathrm{N}$ & $\%$ & $\mathrm{~N}$ & $\%$ & $\mathrm{~N}$ & $\%$ & $\mathrm{~N}$ & $\%$ & $\mathrm{~N}$ & $\%$ \\
\hline Noticia & 52 & 70.3 & 29 & 54.6 & 17 & 56.67 & 12 & 48 & $\mathbf{1 1 0}$ & $\mathbf{6 0 . 7 7}$ \\
\hline Reportaje & 5 & 6.8 & 3 & 5.7 & 0 & 0.00 & 2 & 8 & $\mathbf{1 0}$ & $\mathbf{5 . 5 2}$ \\
\hline Perfil/Entrevista & 1 & 1.4 & 6 & 11.3 & 0 & 0.00 & 6 & 24 & $\mathbf{1 3}$ & $\mathbf{7 . 1 8}$ \\
\hline Perfil & 9 & 12.1 & 3 & 5.7 & 8 & 26.67 & 0 & 0 & $\mathbf{2 0}$ & $\mathbf{1 1 . 0 5}$ \\
\hline Artículo de opinión & 2 & 2.7 & 0 & 0 & 0 & 0.00 & 4 & 16 & $\mathbf{6}$ & $\mathbf{3 . 3 1}$ \\
\hline Carta al director & 1 & 1.4 & 0 & 0 & 0 & 0.00 & 0 & 0 & $\mathbf{1}$ & $\mathbf{0 . 5 5}$ \\
\hline Nota breve & 3 & 4.1 & 12 & 22.6 & 5 & 16.67 & 1 & 4 & $\mathbf{2 1}$ & $\mathbf{1 1 . 6 0}$ \\
\hline TOTAL & 73 & 100 & 53 & 100 & 30 & 100 & 25 & 100 & $\mathbf{1 8 1}$ & $\mathbf{1 0 0}$ \\
\hline
\end{tabular}

Fuente: elaboración propia

Observando los géneros periodísticos utilizados por el diario El Mercurio, que encabeza la lista al ser el medio que más unidades de análisis posee, se evidencia que centró su cobertura electoral priorizando lo informativo, al tener el porcentaje más alto de uso del género noticia: $70.3 \%$.

Con el 12.1\%, el perfil fue el segundo género más utilizado, aunque es necesario puntualizar que de los nueve perfiles publicados durante la campaña electoral por El Mercurio, apenas uno estuvo centrado en algún candidato a la alcaldía, cuatro en candidatos a concejales e igual número en ciudadanos no participantes en el proceso electoral que mencionaron a los postulantes a la alcaldía de Manta; es decir, estas unidades de análisis no contribuyeron en mayor medida al conocimiento de los seis candidatos a burgomaestres.

El reportaje -un género que permite profundizar y ampliar la información- representa tan solo el $6.8 \%$, lo que refleja la poca profundización en la investigación periodística, una tendencia que también fue identificada por Peñaherrera Pozo (2013).

Es considerable la baja presencia de artículos de opinión donde se hizo mención a los candidatos a la alcaldía de Manta, el 2.7\% de presencia indica que los articulistas del medio evitaron pronunciarse sobre los comicios electorales. Otro género de baja presencia fue el perfil/entrevista, que apenas apareció en una ocasión $(1,4 \%)$ durante el periodo de estudio, lo que indica que los candidatos tuvieron siempre una representación mediada por los periodistas que elaboraron las notas.

Diario La Marea mantuvo la tendencia de priorizar la cobertura informativa, con la noticia como el género más común con un $54.6 \%$, es decir, una de cada dos unidades de análisis corresponde a este género. Fiel a su diseño, La Marea fue el medio que más notas breves emitió, 
con un 22,6\%: estos espacios cortos permitieron difundir fugazmente hechos menos relevantes de la campaña electoral. En el medio citado, el perfil/entrevista fue aplicado en una ocasión por cada candidato, con lo que obtuvo un 11.3\%; este género combinó la tradicional entrevista con datos personales, presentando así una información más completa al lector. El perfil fue un género destinado a los candidatos a concejales, quienes hicieron mención de los aspirantes a la alcaldía por sus respectivos partidos, ocupando un 5.7\%. No se difundieron cartas al editor y artículos de opinión donde se mencionaron a los candidatos a la alcaldía, lo que concuerda con el reducido espacio que diariamente ocupa la sección editorial en La Marea.

Diario El Manaba fue el medio que menos géneros utilizó, dejando a un lado los perfiles/entrevistas, artículos de opinión, reportajes y cartas al editor. De los tres géneros aplicados, la noticia nuevamente encabeza la lista con el 56.67\%, manteniendo así la tendencia de cobertura informativa; el $22.67 \%$ corresponde a perfiles realizados tanto a candidatos a la alcaldía como a concejales y ciudadanos.

El Diario Manabita fue el medio que tuvo mayor presencia de géneros como el reportaje, el perfil/entrevista y los artículos de opinión; sin embargo, esto no impidió que, tal como se registró en los otros tres medios, la noticia sea el género predominante con un $48 \%$. Al igual que La Marea (perteneciente a la misma empresa), este medio dedicó un perfil/entrevista a cada candidato, acaparando un $24 \%$ de las unidades de análisis; mientras que el $16 \%$ corresponde a artículos de opinión.

El uso de los géneros en El Diario Manabita parece haber estado marcado temporalmente, ya que las noticias tuvieron mayor protagonismo al inicio y al final de la campaña; los cuatro artículos de opinión publicados aparecieron entre el 10 y el 27 de enero, y los seis perfiles/entrevistas entre el 29 de enero y el 4 de febrero.

\subsubsection{Temas centrales de las unidades}

Los resultados obtenidos en cuanto a los temas centrales abordados en las unidades de análisis, nos indican que las actividades de campaña tuvieron un rol preponderante en la cobertura informativa de los medios de comunicación local durante la campaña por la alcaldía de Manta del 2014, con un $45.05 \%$. La mención u opinión vertida sobre algún candidato fue el siguiente tópico más relevante, con un $18.68 \%$; mientras que las propuestas de campaña acapararon el $14.29 \%$

En diario El Mercurio las actividades de campaña y la opinión/mención de los candidatos, tuvo un rango de $32.9 \%$ cada uno; por lo que se deduce que la parte informativa de la cotidianidad electoral y la opinión de diversos sectores sobre los candidatos fueron base de la información vertida por este medio. 
FIG. 2 Temas de los medios estudiados durante las elecciones a la alcaldía de Manta (Ecuador) en 2014

\begin{tabular}{|c|c|c|c|c|c|c|c|c|c|c|}
\hline \multirow{2}{*}{ Tema central } & \multicolumn{2}{|c|}{$\begin{array}{l}\text { E1 } \\
\text { Mercurio }\end{array}$} & \multicolumn{2}{|c|}{$\begin{array}{l}\text { La } \\
\text { Marea }\end{array}$} & \multicolumn{2}{|c|}{$\begin{array}{l}\text { E1 } \\
\text { Manaba }\end{array}$} & \multicolumn{2}{|c|}{$\begin{array}{l}\text { E1 } \\
\text { Diario }\end{array}$} & \multicolumn{2}{|c|}{ Total } \\
\hline & $\mathrm{N}$ & $\%$ & $\mathrm{~N}$ & $\%$ & $\mathrm{~N}$ & $\%$ & $\mathrm{~N}$ & $\%$ & $\mathrm{~N}$ & $\%$ \\
\hline $\begin{array}{ll}\begin{array}{l}\text { Actividades } \\
\text { campaña }\end{array} & \text { de }\end{array}$ & 24 & 32.9 & 34 & 64.2 & 16 & 51.6 & 8 & 32 & 82 & $\begin{array}{l}45.3 \\
0\end{array}$ \\
\hline $\begin{array}{ll}\text { Propuestas } & \text { de } \\
\text { campaña } & \end{array}$ & 4 & 5.5 & 9 & 17 & 7 & 22.6 & 6 & 24 & 26 & $\begin{array}{l}14.3 \\
6\end{array}$ \\
\hline El candidato/Perfil & 1 & 1.4 & 0 & 0 & 1 & 3.2 & 0 & 0 & 2 & 1.10 \\
\hline Opinión/Mención & 24 & 32.9 & 3 & 5.7 & 3 & 9.7 & 4 & 16 & 34 & $\begin{array}{l}18.7 \\
8\end{array}$ \\
\hline Respaldo & 9 & 12.3 & 1 & 1.9 & 0 & 0.0 & 0 & 0 & 10 & 5.52 \\
\hline Otro & 11 & 15.1 & 6 & 11.3 & 3 & 12.9 & 7 & 28 & 27 & $\begin{array}{l}14.9 \\
2\end{array}$ \\
\hline TOTAL & 73 & 100 & 53 & 100 & 30 & 100 & 25 & 100 & $\begin{array}{l}18 \\
1\end{array}$ & 100 \\
\hline
\end{tabular}

Fuente: elaboración propia

Un hecho que no pasa desapercibido es que el 12.3\% de la información difundida por este medio se centró en el respaldo explícito concedido a Jaime Estrada, privilegio que no tuvo ningún otro candidato a la alcaldía de Manta. Asimismo, diario El Mercurio fue el medio que menor porcentaje designó a las propuestas de campaña como tema central con apenas un $5.5 \%$.

Con un $64.2 \%$, las actividades campaña fueron el centro de la información difundida por el diario La Marea, siendo este el medio que más alto porcentaje obtuvo frente a esta temática. Estas actividades se complementaron con las propuestas realizadas por los candidatos, que tuvieron un $17 \%$ de predominancia.

Diario El Manaba mantuvo una tendencia similar a la de La Marea, mostrando un decrecimiento de las actividades de campaña y un incremento en las propuestas de los candidatos, alcanzando un $51.6 \%$ y $22.6 \%$, respectivamente.

El Diario fue el medio que presentó un mayor equilibrio en cuanto a los temas centrales, registrando el índice más bajo de actividades electorales con un $32 \%$, y el más alto de propuestas de campaña con un $24 \%$.

El índice registrado como otros responde principalmente a hechos informativos ajenos al quehacer electoral y demás hechos que no lograron encajar en las otras cinco opciones. 


\section{1. 3 Secciones utilizadas}

En este aspecto, los medios de comunicación presentaron dos tendencias, la primera de los diarios El Mercurio y El Manaba, donde predominó la sección local; y la segunda, representada por los medios de Ediasa, que utilizaron una sección creada especialmente para difundir la información referente a la campaña electoral, tal como se aprecia en la siguiente tabla:

FIG. 3 Secciones utilizadas por los medios escritos durante las elecciones a la alcaldía de Manta (Ecuador) en 2014

\begin{tabular}{|l|l|l|l|l|l|l|l|l|l|l|}
\hline \multirow{2}{*}{ Sección } & \multicolumn{2}{|l|}{ E1 Mercurio } & \multicolumn{2}{l|}{ La Marea } & \multicolumn{2}{l}{ E1 Manaba } & \multicolumn{2}{l|}{ E1 Diario } & \multicolumn{2}{l|}{ Total } \\
\cline { 2 - 12 } & $\mathrm{N}$ & $\%$ & $\mathrm{~N}$ & $\%$ & $\mathrm{~N}$ & $\%$ & $\mathrm{~N}$ & $\%$ & $\mathrm{~N}$ & $\%$ \\
\hline Local & 44 & 60.3 & 0 & 0 & 18 & 60.0 & 1 & 4.00 & $\mathbf{6 3}$ & $\mathbf{3 4 . 8 1}$ \\
\hline Provincial & 2 & 2.7 & 0 & 0 & 0 & 0.0 & 0 & 0.00 & $\mathbf{2}$ & $\mathbf{1 . 1 0}$ \\
\hline Sección elecciones & 6 & 8.2 & 48 & 90.6 & 7 & 23.3 & 19 & 76.00 & $\mathbf{8 0}$ & $\mathbf{4 4 . 2 0}$ \\
\hline Sociales/farándula & 4 & 5.5 & 0 & 0 & 1 & 3.3 & 0 & 0.00 & $\mathbf{5}$ & $\mathbf{2 . 7 6}$ \\
\hline Opinión & 4 & 5.5 & 0 & 0 & 0 & 0.0 & 4 & 16.00 & $\mathbf{8}$ & $\mathbf{4 . 4 2}$ \\
\hline Deportes & 3 & 4.1 & 0 & 0 & 3 & 10.0 & 1 & 4.00 & $\mathbf{7}$ & $\mathbf{3 . 8 7}$ \\
\hline No especifica & 2 & 2.7 & 0 & 0 & 0 & 0.0 & 0 & 0.00 & $\mathbf{2}$ & $\mathbf{1 . 1 0}$ \\
\hline Actualidad & 8 & 11 & 2 & 3.8 & 1 & 3.3 & 0 & 0.00 & $\mathbf{1 1}$ & $\mathbf{6 . 0 8}$ \\
\hline Política & 0 & 0 & 3 & 5.7 & 0 & 0.0 & 0 & 0.00 & $\mathbf{3}$ & $\mathbf{1 . 6 6}$ \\
\hline TOTAL & 73 & 100 & 53 & 100 & 30 & 100 & 25 & 100 & $\mathbf{1 8 1}$ & $\mathbf{1 0 0}$ \\
\hline
\end{tabular}

Fuente: elaboración propia

\subsection{Análisis de contenido informatizado}

Los resultados obtenidos como parte del análisis de contenido informatizado nos brindaron más detalles respecto a la cobertura periodística de las elecciones por la alcaldía de Manta en 2014; a nivel macro, buscábamos identificar los elementos temáticos que caracterizaron, cuantitativamente, la representación de las campañas. En la siguiente tabla se presentan las palabras más repetidas en el conjunto global de los cuatro medios analizados:

FIG. 4 Tabla léxica de palabras con mayor frecuencia de los medios escritos durante las elecciones a la alcaldía de Manta (Ecuador) en 2014

\begin{tabular}{|l|l|l|}
\hline Puesto & Palabra & N \\
\hline 1 & Jaime Estrada & 257 \\
\hline
\end{tabular}




\begin{tabular}{|c|c|c|}
\hline 2 & Proyectos & 121 \\
\hline 3 & Agua & 114 \\
\hline 4 & Recorridos & 111 \\
\hline 5 & Propuestas & 104 \\
\hline 6 & Campaña & 100 \\
\hline 7 & Obras & 96 \\
\hline 8 & Jorge Zambrano & 85 \\
\hline 9 & Trabajo & 85 \\
\hline 10 & Apoyo & 76 \\
\hline 11 & Reelección & 75 \\
\hline 12 & Construir & 62 \\
\hline 13 & Casa & 61 \\
\hline 14 & Contaminación & 59 \\
\hline 15 & Plan & 56 \\
\hline 16 & Seguridad & 54 \\
\hline 17 & Respaldo & 52 \\
\hline 18 & Construcción & 50 \\
\hline 19 & Recursos & 47 \\
\hline 20 & Solución & 47 \\
\hline 21 & Mercado & 39 \\
\hline
\end{tabular}

Fuente: elaboración propia

La palabra identificada como la de mayor frecuencia responde al nombre de un candidato: Jaime Estrada. Su posición indica su elevada exposición mediática durante la campaña electoral, al figurar en la tabla léxica con una amplia ventaja sobre las otras palabras, incluso en relación con los demás postulantes, la mayoría de los cuales no figuran en este primer cuadro. En el puesto once aparece una palabra directamente ligada con este aspirante: reelección.

Las dos siguientes palabras: proyectos y agua están vinculadas con las propuestas realizadas por los candidatos, y en particular con dos de los problemas históricos que ha sufrido la población mantense: la falta de líquido vital, tal como lo menciona Othon Castillo (1965) en su obra Sed en el puerto, y la contaminación ambiental (Moreira y Mendoza, 2008) que afecta a amplios sectores como la parroquia Los Esteros, Tarqui, Playita Mía y las zonas aledañas a los cauces fluviales (Expreso, 2014, 8 de febrero). 
En este punto se evidencia que el agua fue un tema relevante en el discurso de los candidatos, quienes manifestaron propuestas para ampliar la cobertura de líquido, principalmente a los barrios periféricos, e implementar acciones contra la contaminación ambiental. Otros ejes temáticos en el mensaje político fueron las obras, orientadas a mejorar el ornato e infraestructura de la urbe; trabajo, como una acción comprometida de los aspirantes a la alcaldía; casas, una propuesta que buscó calar en los votantes atendiendo el déficit de viviendas de interés social; seguridad, una respuesta a un problema que ha tenido índices alarmantes en años anteriores en la ciudad (El Universo, 2011, 29 de diciembre); la solución a las necesidades de la colectividad mantense y la atención al mercado de la parroquia Tarqui, el nervio comercial de la ciudad, y que carece de infraestructura adecuada.

Estos temas vinculados a las ofertas de los seis candidatos, estuvieron acompañados de la difusión de las actividades cotidianas de la campaña electoral, así lo evidencia la presencia de palabras como recorridos, campaña, respaldo y apoyo, que reflejan la cotidianidad de las jornadas previas a las elecciones, donde los partidos y movimientos políticos ponen a trabajar toda su maquinaria para ganar la adhesión de los ciudadanos.

En el puesto ocho, figura el nombre de Jorge Zambrano, quien es el segundo y último candidato que se muestra en esta tabla. Aunque su posición está muy distante de Estrada, su exposición mediática fue mucho más amplia que la de los otros cuatro candidatos que no aparecen entre las palabras con mayor frecuencia en las unidades de análisis.

Para diferenciar las tendencias internas de los cuatro medios, a continuación se muestra una tabla léxica con los resultados desglosados:

FIG. 5 Tabla léxica de palabras con mayor frecuencia de los medios escritos durante las elecciones a la alcaldía de Manta (Ecuador) en 2014

\begin{tabular}{|c|c|c|c|c|c|c|c|}
\hline \multicolumn{2}{|l|}{ E1 Mercurio } & \multicolumn{2}{|l|}{ La Marea } & \multicolumn{2}{|l|}{ E1 Manaba } & \multicolumn{2}{|l|}{ E1 Diario } \\
\hline Palabra & $\mathbf{N}$ & Palabra & $\mathbf{N}$ & Palabra & $\mathbf{N}$ & Palabra & $\mathbf{N}$ \\
\hline Jaime Estrada & $\begin{array}{l}16 \\
6\end{array}$ & $\begin{array}{l}\text { Jorge } \\
\text { Zambrano }\end{array}$ & $\begin{array}{l}5 \\
0\end{array}$ & Jaime Estrada & $\begin{array}{l}1 \\
9\end{array}$ & Agua & $\begin{array}{l}3 \\
4\end{array}$ \\
\hline Proyectos & 72 & Jaime Estrada & $\begin{array}{l}4 \\
7\end{array}$ & $\begin{array}{l}\text { Jorge } \\
\text { Zambrano }\end{array}$ & $\begin{array}{l}1 \\
6\end{array}$ & Campaña & $\begin{array}{l}2 \\
8\end{array}$ \\
\hline Trabajo & 53 & Campaña & $\begin{array}{l}4 \\
6\end{array}$ & Recorridos & $\begin{array}{l}1 \\
6\end{array}$ & Jaime Estrada & $\begin{array}{l}2 \\
5\end{array}$ \\
\hline Propuestas & 51 & Recorridos & $\begin{array}{l}4 \\
2\end{array}$ & Propuestas & $\begin{array}{l}1 \\
1\end{array}$ & Obras & $\begin{array}{l}1 \\
8\end{array}$ \\
\hline Apoyo & 49 & Agua & $\begin{array}{l}3 \\
7\end{array}$ & Obras & $\begin{array}{l}1 \\
0\end{array}$ & $\begin{array}{l}\text { Contaminació } \\
\mathrm{n}\end{array}$ & $\begin{array}{l}1 \\
7\end{array}$ \\
\hline Obras & 48 & Propuestas & $\begin{array}{l}2 \\
9\end{array}$ & Proyectos & 8 & Solución & $\begin{array}{l}1 \\
7\end{array}$ \\
\hline
\end{tabular}




\begin{tabular}{|c|c|c|c|c|c|c|c|}
\hline Reelección & 47 & Proyectos & $\begin{array}{l}2 \\
7\end{array}$ & Trabajo & 8 & Recorridos & $\begin{array}{l}1 \\
6\end{array}$ \\
\hline Respaldo & 42 & Contaminación & $\begin{array}{l}2 \\
4\end{array}$ & Caravana & 7 & Proyectos & $\begin{array}{l}1 \\
4\end{array}$ \\
\hline Construcción & 37 & Solución & $\begin{array}{l}2 \\
3\end{array}$ & Contaminación & 7 & Plan & $\begin{array}{l}1 \\
3\end{array}$ \\
\hline Agua & 37 & Recursos & $\begin{array}{l}2 \\
2\end{array}$ & Agua & 6 & Propuestas & $\begin{array}{l}1 \\
3\end{array}$ \\
\hline Seguridad & 37 & Casas & $\begin{array}{l}2 \\
2\end{array}$ & Campaña & 6 & Recursos & $\begin{array}{l}1 \\
3\end{array}$ \\
\hline Recorridos & 37 & Apoyo & $\begin{array}{l}2 \\
2\end{array}$ & Reelección & 6 & $\begin{array}{l}\text { Jorge } \\
\text { Zambrano }\end{array}$ & $\begin{array}{l}1 \\
2\end{array}$ \\
\hline Casas & 26 & Obras & $\begin{array}{l}2 \\
0\end{array}$ & $\begin{array}{l}\text { María } \\
\text { Zambrano }\end{array}$ & 5 & Casas & $\begin{array}{l}1 \\
2\end{array}$ \\
\hline Mercado & 25 & $\begin{array}{l}\text { Gustavo } \\
\text { Andrade }\end{array}$ & $\begin{array}{l}1 \\
9\end{array}$ & Encuestas & 5 & Tratamiento & 9 \\
\hline Rafael Correa & 22 & Trabajo & $\begin{array}{l}1 \\
9\end{array}$ & Plan & 5 & Denuncia & 9 \\
\hline Regeneración & 21 & Caravana & $\begin{array}{l}1 \\
8\end{array}$ & $\begin{array}{l}\text { Juan } \quad \text { Carlos } \\
\text { Alvia }\end{array}$ & 4 & Planta de Agua & 8 \\
\hline Plan & 20 & Plan & $\begin{array}{l}1 \\
8\end{array}$ & Problemas & 4 & Paso elevado & 7 \\
\hline Campaña & 20 & $\begin{array}{l}\text { María } \\
\text { Zambrano }\end{array}$ & $\begin{array}{l}1 \\
7\end{array}$ & Apoyo & 4 & EPAM & 7 \\
\hline Tratamiento & 16 & Terreno & $\begin{array}{l}1 \\
7\end{array}$ & Respaldo & 4 & $\begin{array}{l}\text { Gustavo } \\
\text { Andrade }\end{array}$ & 7 \\
\hline $\begin{array}{l}\text { Mariano } \\
\text { Zambrano }\end{array}$ & 14 & $\begin{array}{ll}\text { Juan } & \text { Carlos } \\
\text { Alvia } & \end{array}$ & $\begin{array}{l}1 \\
6\end{array}$ & Mercado & 3 & Regeneración & 7 \\
\hline Gestión & 13 & $\begin{array}{l}\text { Leonardo } \\
\text { Cárdenas }\end{array}$ & $\begin{array}{l}1 \\
6\end{array}$ & $\begin{array}{l}\text { Plan } \\
\text { Hidrosanitario }\end{array}$ & 3 & Deuda & 7 \\
\hline
\end{tabular}

Fuente: elaboración propia

Los contenidos emitidos por diario El Mercurio tuvieron como personaje central al candidato Jaime Estrada, quien figura en el primer lugar con 166 menciones, una cifra que está muy por encima de cualquier otro término registrado en las unidades de análisis sobre la campaña electoral.

A la figura del candidato oficialista le acompañaron términos como apoyo, respaldo y reelección, lo que nos brinda una idea del direccionamiento que tuvo el discurso periodístico de este medio, reflejando un constante sobredimensionamiento de la aceptación ciudadana. 
Los términos proyectos, propuestas, obras y plan denotan la relevancia que tuvieron las ofertas del candidato en el discurso del diario El Mercurio; estas acciones básicamente estuvieron enmarcadas en aspectos como agua, seguridad, casas, mercado, tratamiento de aguas residuales y regeneración.

Este discurso periodístico también vinculó a la figura de Jaime Estrada con la del presidente de la República (Rafael Correa) y la del prefecto de Manabí (Mariano Zambrano), ambos personajes se erigen como líderes a nivel nacional y provincial respectivamente, por lo cual se pretendió crear una actitud positiva de los votantes hacia el candidato, asociándolo con estos dos personajes.

Respecto a diario La Marea se aprecia en los resultados una alta exposición de los candidatos Jorge Zambrano con 50, y Jaime Estrada con 47 menciones; además, los otros cuatro candidatos aparecen entre las palabras más frecuentes: Gustavo Andrade con 19, María Zambrano con 17, y Juan Carlos Alvia y Leonardo Cárdenas con 16. A simple vista destaca la paridad que existió entre los dos primeros candidatos, y entre los cuatro últimos, marcando así dos grupos plenamente identificados de acuerdo a las menciones.

Diario La Marea fue el único medio donde Zambrano tuvo mayor exposición mediática, y también donde aparecen los seis candidatos en la lista de las palabras más frecuentes de acuerdo al análisis de contenido informatizado.

Las palabras campaña y recorridos figuran en la tercera y cuarta posición respectivamente, lo que confirma el protagonismo que tuvo el desarrollo de las actividades proselitistas en la agenda mediática de La Marea. El agua es la figura principal dentro de los planteamientos realizados por los candidatos en el diario La Marea, manteniendo así la tendencia registrada en los demás medios. El término contaminación aparece como una de las principales problemáticas de la ciudad, y frente a la cual los candidatos tuvieron que presentar sus propuestas de solución.

La aparición del término recursos implica el trato del tema presupuestario que sustente las propuestas, así como la sostenibilidad de la administración municipal, lo que denota un trato un poco más profundo en estos aspectos. Una propuesta que tuvo un relativo protagonismo frente a los otros medios, fue el de la entrega de terrenos a personas de escasos recursos, acción que nuevamente toma como protagonistas a los candidatos Jaime Estrada y Jorge Zambrano, quienes sacaron a la luz sus proyectos de venta de lotes a bajo costo.

En el diario El Manaba, nuevamente Estrada y Zambrano figuran a la cabeza del listado con 19 y 16 menciones respectivamente. El marco en el que se desarrolló la cobertura periodística fueron las actividades proselitistas, figurando términos como recorridos, caravana, campaña, apoyo y respaldo. Mientras que en lo concerniente a propuestas y proyectos se mantienen aspectos como la contaminación, agua, mercado y plan hidrosanitario. Un término diferenciador en ElManaba es encuestas, que figura en cinco ocasiones, marcando así el protagonismo que le brindó este medio a los sondeos realizados por Cedatos y Opecuador de Santiago Pérez, donde se ponía a dos candidatos como posibles ganadores de la alcaldía de Manta. María Zambrano y Juan Carlos Alvia fueron los otros dos candidatos que figuran en esta lista con cinco y cuatro menciones 
cada uno, teniendo así una cobertura inferior a la de Estrada y Zambrano, pero mayor que la de Cárdenas y Andrade.

El único medio donde en primer lugar figura una palabra que no es el nombre de algún candidato es el Diario Manabita, donde aparece la palabra agua como la de mayor presencia, a lo que se suman otros términos íntimamente ligados como planta de agua y EPAM (Empresa Pública Aguas de Manta) con ocho y siete menciones respectivamente, quedando claro que los problemas y propuestas vinculadas a la dotación de líquido vital fue el tema central de la cobertura periodística de este medio en las elecciones de Manta en el 2014. Pero esta no es la única particularidad que presenta el Diario Manabita, ya que también se muestran por primera vez otros términos que denotan un mayor contenido en las unidades de análisis. Uno de ellos es recursos, con trece menciones, denotando una relativa importancia al posible financiamiento de las propuestas y a la situación económica del cabildo local. Asimismo, la palabra deuda fue una de las protagonistas en este medio, haciendo alusión a los cuestionamientos del supuesto sobreendeudamiento manejado por la entonces administración municipal, y el dilema por la aprobación del crédito otorgado por el Banco Mundial (por un valor de cien millones de dólares), lo que puso en el tapete del debate electoral el manejo de esos recursos y si el cabildo estaba en capacidad de asumir esa responsabilidad económica (El Diario, 2014, 13 de febrero). En esta misma tendencia figura denuncia, un hecho que tuvo como principales protagonistas a los movimientos políticos de los candidatos Zambrano y Estrada, e incluyó la protesta por supuestos daños en las publicidades electorales y prácticas proselitistas no permitidas. Respecto a los candidatos, Jaime Estrada figura en la tercera posición con veinticinco menciones, Jorge Zambrano con doce menciones en la doceava casilla, y Gustavo Andrade con siete menciones en la decimonovena.

\subsection{Entrevistas en profundidad}

De acuerdo a la percepción de los candidatos, el rol de los medios de comunicación durante las elecciones 2014, estuvo marcado por la inequidad. Juan Carlos Alvia indicó un trato preferencial hacia los políticos respaldados por el poder y el factor económico. Gustavo Andrade reconoció el interés de La Marea y El Diario Manabita por actuar con equidad, contrariamente señaló a los dos medios restantes de tomar abiertamente partido por la desinformación. Para Leonardo Cárdenas los medios definieron sus posturas editoriales o noticias influenciados por su afinidad política u otros tipos de intereses. Mientras que María Zambrano definió los tratamientos electorales desplegados por estos medios con una sola palabra: parcializados.

La percepción de los candidatos frente a la influencia que tuvieron los medios de comunicación es diversa, Juan Carlos Alvia y Gustavo Andrade indicaron que la influencia fue evidente, favoreciendo a los candidatos más expuestos positivamente por los medios; mientras que Leonardo Cárdenas señaló que al ser una parcialización tan evidente se generó un efecto boomerang en contra de uno de los candidatos; María Zambrano, por su parte, manifestó que no se debe exagerar respecto al poder influencia de los medios de comunicación. Estas impresiones de los candidatos, según puede deducirse, subrayan el tratamiento descompensado que también ha sido comprobado a través de las técnicas cuantitativas. 


\section{Conclusiones}

Una vez descritos y analizados los resultados obtenidos como parte de la presente investigación, sí se logró comprobar la hipótesis planteada:

La emisión de contenidos digitales difundidos por los medios de comunicación escritos de Manabi durante la campaña electoral por la alcaldía de Manta en 2014, favoreció la exposición mediática de determinados candidatos.

Según ha podido observarse a través de las distintas técnicas investigativas, las coberturas informativas brindadas durante la campaña electoral por la alcaldía de Manta estuvieron centradas mayoritariamente en el aspecto informativo de las actividades proselitistas. Los medios objeto de estudio no mostraron una cultura de análisis y opinión para orientar a la ciudadanía hacia reflexiones profundas respecto a las propuestas de los candidatos. En los medios El Mercurio y El Manaba se evidenció una falta de planificación en la estructura de la información concerniente a la campaña electoral.

En general, se ha anotado una clara tendencia a favor de los candidatos Jaime Estrada y Jorge Zambrano, en desmedro de los candidatos Juan Carlos Alvia, Gustavo Andrade, Leonardo Cárdenas y María Zambrano, con lo que se inobservó el Art. 72 de la Ley Orgánica de Comunicación, el cual asegura que todos los participantes en los procesos electorales deben contar con un trato equitativo. Desde la academia o desde los organismos de control se deberían generar espacios donde la ciudadanía aprenda a consumir los contenidos de los medios de comunicación de una manera más analítica, facilitando así la identificación de discursos periodísticos direccionados o sesgados. En ese sentido, sería recomendable establecer estrategias conjuntas para favorecer un tratamiento más equitativo de los candidatos electorales, es decir, priorizar los contenidos centrados en los perfiles, propuestas y proyectos de los candidatos en condiciones de equidad.

En futuros trabajos, resultaría conveniente replicar las técnicas empleadas sobre otros fenómenos electorales, como por ejemplo la elección de la Presidencia de la República, para comprobar hasta qué punto se dan los efectos señalados en medios de orientación nacional. También hubiese sido interesante aplicar una encuesta a la propia ciudadanía, por cuanto las transformaciones comunicacionales que se han dado en Ecuador -con la aprobación, entre otras, de la Ley Orgánica de Comunicación en 2013- tienden a incorporar activamente a la sociedad dentro de su relación con los medios de comunicación. Y sus percepciones, por tanto, hubiesen ayudado enormemente a interpretar si existe un distanciamiento social con respecto de las coberturas analizadas.

Todas estas tareas, en cualquier caso, nos las fijamos como líneas posibles de investigación para un objeto y para un contexto en que, como decíamos páginas atrás, se ha escrito muy poco. 


\section{Bibliografía}

Abad, G. (2011). El Club de la pelea. Gobierno y medios, un entramado de fuerzas y debilidades. Quito: Ciespal.

Abela, J. A. (2002). Las técnicas de análisis de contenido: Una revisión actualizada. Granada: Universidad de Granada.

Allport, F. (1937). Toward a science of public opinion. Public Opinion Quarterly, 1(1), 7-23.

Alonso, I. F. y Santana, F. (2000). Estado y medios de comunicación en la España democrática. Madrid: Alianza.

Barredo, D.; Oller, M. y Buenaventura, S. (2013). The End of the Information Society. Notes for the Configuration of the New Contemporary Public Space: the Society of Devisers. Observatorio (OBS*), 7(3), 079 - 091.

Barredo, D. y Silva, R. (2014). Interacción institucional, participación ciudadana y transparencia. La iniciativa del Voto Transparente en el Ecuador (2013 - 2014). Chasqui, Revista Latinoamericana de Comunicación, 126, 4-13.

Barredo, D.; Pérez Zúñiga, J. M.; Vivas, R.; y Fernández, M. (2015). "El periodismo ecuatoriano en entredicho. Descenso de credibilidad y nuevas perspectivas comunicacionales". En Barredo, D.; Oller, M. y Hernández, S. (Eds.). La Comunicación y el Periodismo de Ecuador frente a los desafíos contemporáneos <pp. 107 - 126>. La Laguna: Sociedad Latina de Comunicación Social.

Berelson, B. (1952). Content Analysis in Communication Research. Glencoe, Illinois: The Free Press.

Boczkowski, P. J. (2006). Digitalizar las noticias. Buenos Aires: Manantial.

Castells, M. (2009). Comunicación y poder. Madrid: Alianza.

Castillo, O. (1965). Sed en el puerto. Quito: Casa de la Cultura Ecuatoriana.

De Fleur, L. y Ball-Rokeach, S. J. (1988). Teorias de la comunicación de masas. México: Paidós.

El Diario (2014, 13 de febrero). Gustavo Andrade: "El endeudamiento es innecesario". Consultado el 30/03/2015 de: http://www.eldiario.ec/noticias-manabi-ecuador/305072gustavo-andrade-el-endeudamiento-es-innecesario/

El Universo (2011, 29 de diciembre). Taxista es víctima 103 de la violencia e inseguridad en Manta en el 2011. Consultado el 30/03/2015 de: http://www.eluniverso.com/2011/12/29/1/1422/taxista-victima-103-2011-violenciainseguridad-manta.html

El Universo (2012, 11 de noviembre). 'Viejos' y 'famosos' buscan más curules. Consultado el 30/03/2015 de: http://www.eluniverso.com/2012/11/11/1/1355/viejos-famosos-buscanmas-curules.html

eleccionesenecuador.com (s. f. ). Elecciones 2014 Cantón Manta - provincia Manabí. Consultado el 30/03/2015 de: http://www.eleccionesenecuador.com/elecciones-cantonMANTA-provincia-manabi-132.html

Expreso (2014, 8 de febrero). La contaminación, el lío de Manta. Consultado el 30/03/2015 de: $\underline{\text { http: } / / \text { expreso.ec/expreso/plantillas/nota print.aspx?idArt }=5703751 \& \text { tipo }=2}$ 
Fraser, N. (1999). Repensando la esfera pública: una contribución a la crítica de la democracia actualmente existente. Revista Ecuador Debate, 46, 139-174.

Goffman, E. y Rodríguez, J. L. (2006). Frame analysis: los marcos de la experiencia. Madrid: Centro de Investigaciones Sociológicas.

Habermas, J. (1988). La esfera de lo público. Revista del Instituto de Investigaciones sociales de Montevideo, 123-130.

Hernández Sampieri, R.; Fernández, C. y Baptista, P. (2010). Metodología de la investigación. México: McGraw Hill.

Igartua, J. J. y Muñiz, C. (2012). Encuadres noticiosos e inmigración: un análisis de contenido de la prensa y televisión españolas. Zer: Revista de Estudios de Comunicación, 9(16).

Isch, G. (2012). De la guerra de guerrillas a la guerra de cuartillas. La comunicación en el gobierno de la revolución ciudadana. Quito: Ciespal.

León, O. (2007). Comunicación Política: Ecuador: Elecciones, medios y democracia. Chasqui, Revista Latinoamericana de Comunicación, 97, 50-55.

Lull, J. (1997). Medios, comunicación, cultura: aproximación global. Buenos Aires: Amorrortu.

Ministerio de Telecomunicaciones (2014, 19 de mayo). El 40,4\% de los Ecuatorianos utilizó internet en los últimos 12 meses. Recuperado el 1 de diciembre de 2014 de http://www.telecomunicaciones.gob.ec/el -404-de -los - ecuatorianos - utilizo -internet -en -los -ultimos -12 -meses

McQuail, D. (2000). Introducción a la teoría de la comunicación de masas <tercera edición>. Barcelona: Paidós.

Moreira, J. A. y Mendoza, R. (2008). Procesos de participación ciudadana con enfoque de equidad social y de género en la problemática de la contaminación ambiental producida por las industrias en la Parroquia "Los Esteros" del Cantón Manta y sus áreas de influencia, año 2006-2007<tesis de grado>. Ambato: Universidad Técnica de Ambato.

Muñoz, B. (2005). Modelos culturales: teoría sociopolítica de la cultura <Vol. 152>. Barcelona: Anthropos.

Noelle - Neumann, E. (1995). La espiral del silencio. Barcelona: Paidós.

Oller, M. y Barredo, D. (2012). La Sociedad de los Ideantes: Repensando los conceptos de opinión y esfera pública y las teorías democráticas relacionadas con el fenómeno comunicativo ciudadano. Tenerife: Sociedad Latina de Comunicación Social. Consultado el 07/05/2014 de: http://www.revistalatinacs.org/067/cuadernos/29 Oller.pdf

Ortega, F. (2011). La politica Mediatizada. Madrid: Alianza.

Peñaherrera, N. A. (2013). Análisis de la agenda setting de los medios de comunicación: televisivos, impresos y digitales del Ecuador, entre abrily mayo del 2012 (Diario El Mercurio de la ciudad de Manta) < tesis de grado>. Loja: UTPL.

Quivy, R. y Van Campenhoudt, L. (1992). Manual de investigación en ciencias sociales. México DF: Limusa.

Ramonet, I. (2011). La Explosión del Periodismo. Buenos Aires: Capital Intelectual.

Rivera Rogel, J. (2013). Mapa de Medios digitales del Ecuador 2012. Quito: Ciespal. 
Rivera Rogel, J. (2014). Rafael Correa y las elecciones 2006. Inicios del Marketing y Comunicación política digital en Ecuador. Chasqui, Revista Latinoamericana de Comunicación, 126, $116-123$.

Rivera Costales, D. (2011). El ciberperiodismo en Ecuador. Casos de estudio La Hora y Crónica, periódicos de Loja. Quórum Académico, 8(16), 269-280.

Rivera Costales, D. (2011). Evolución del ciberperiodismo en Ecuador a través de los periódicos de Loja. Razón y Palabra, 71.

Ruiz, E. S. (2005). Medios de comunicación y democracia <Vol. 32>, Bogotá: Norma.

Thompson, J. B. (1996). La teoría de la esfera pública. Voces y culturas, 10, 81-96.

\section{Notas}

${ }^{1}$ El presente estudio es una adaptación procedente de la tesis "Los contenidos digitales escritos en los medios de comunicación de Manabí y su relación con la exposición mediática de los candidatos a la alcaldía de Manta (2014)", defendida con la máxima calificación por Juan Pablo Trámpuz en diciembre de 2014 y dirigida por el Dr. Daniel Barredo Ibáñez.

${ }^{2}$ La opinión pública ha tenido las más diversas conceptualizaciones, sin embargo es un término frecuente en los estudios de la comunicación social (Noelle-Neumann, 1995) y cuyo surgimiento ha sido asociado al nacimiento de los medios de comunicación de masas, según interpretamos a Ramonet (2011).

${ }^{3}$ Según datos consultados el 20/01/2014.

${ }^{4}$ Consultado el 20/07/2014 http://cne.gob.ec/index.php/sala-de-prensa1/noticiasanteriores/4991-se-registran-331-ordenes-de-pautaje-de-publicidad-electoral

${ }^{5}$ Consultado el 20/07/2014 http://www.fundamedios.org/autorregulacioncapacitacion/articulos/medios-digitales-aumentan-en-ecuador

${ }^{6}$ Para más información se puede visitar http://www.concordancesoftware.co.uk/ 


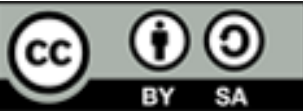

Licencia Creative Commons

Miguel Hernández Communication Journal

mhcj.es

\section{Forma de citar este artículo en las bibliografías}

Juan Pablo Trámpuz y Daniel Barredo Ibáñez (2015): “Tratamiento electoral en ecuador: estudio sobre la exposición mediática de los candidatos a alcalde en las elecciones de Manta (2014)", en Miguel Hernández Communication Journal, nº, páginas 69 a 90. Universidad Miguel Hernández, UMH (Elche-Alicante). Recuperado el _ de _ de 20_ de: http://mhjournal.org/[link del artículo] 